\title{
The Impact of Hospital-Wide Use of a Tapered-Cuff Endotracheal Tube on the Incidence of Ventilator-Associated Pneumonia
}

\author{
David L Bowton MD, R Duncan Hite MD, R Shayn Martin MD, and Robert Sherertz MD
}

\begin{abstract}
BACKGROUND: Aspiration of colonized oropharyngeal secretions is a major factor in the pathogenesis of ventilator-associated pneumonia (VAP). A tapered-cuff endotracheal tube (ETT) has been demonstrated to reduce aspiration around the cuff. Whether these properties are efficacious in reducing VAP is not known. METHODS: This 2-period, investigator-initiated observational study was designed to assess the efficacy of a tapered-cuff ETT to reduce the VAP rate. All intubated, mechanically ventilated patients over the age of 18 were included. During the baseline period a standard, barrel-shaped-cuff ETT (Mallinckrodt Hi-Lo) was used. All ETTs throughout the hospital were then replaced with a tapered-cuff ETT (TaperGuard). The primary outcome variable was the incidence of VAP per 1,000 ventilator days. RESULTS: We included 2,849 subjects, encompassing 15,250 ventilator days. The mean \pm SD monthly VAP rate was $3.29 \pm 1.79$ / 1,000 ventilator days in the standard-cuff group and $2.77 \pm 2.00 / 1,000$ ventilator days in the tapered-cuff group $(P=.65)$. While adherence to the VAP prevention bundle was high throughout the study, bundle adherence was significantly higher during the standard-cuff period $(96.5 \pm 2.7 \%)$ than in the tapered-cuff period $(90.3 \pm 3.5 \%, P=.01)$. CONCLUSIONS: In the setting of a VAP rate very near the average of ICUs in the United States, and where there was high adherence to a VAP prevention bundle, the use of a tapered-cuff ETT was not associated with a reduction in the VAP rate. Key words: ventilator-associated pneumonia; prevention; risk; quality improvement. [Respir Care 2013;58(10):1582-1587. ( 2013 Daedalus Enterprises]
\end{abstract}

\section{Introduction}

Ventilator-associated pneumonia (VAP) is a common nosocomial infection in the ICU. ${ }^{1}$ Five to 20 percent of patients who require mechanical ventilation for $>48$ hours

Dr Bowton is affiliated with the Critical Care Section, Department of Anesthesiology; Dr Hite is affiliated with the Department of Pulmonary, Critical Care, Allergy, and Immunology; Dr Martin is affiliated with the Department of General Surgery; and Dr Sherertz is affiliated with the Department of Infectious Disease, Wake Forest Baptist Health, WinstonSalem, North Carolina.

This research was partly supported by Covidien. See Methods for details. Drs Bowton and Hite were partly supported by grants from the National Institutes of Health. Drs Martin and Sherertz have no disclosed no other conflicts of interest.

Correspondence: David L Bowton MD, Department of Anesthesiology, Wake Forest Baptist Health, Medical Center Boulevard, Winston-Salem NC 27157-1009. E-mail: dbowton@wakehealth.edu.

DOI: $10.4187 /$ respcare. 02278 will develop VAP, and the associated mortality is 15$50 \% .^{2,3}$ ICU stay in patients with VAP is increased by a mean of 6.1 days, and the excess cost can be as high as $\$ 40,000$ per patient. ${ }^{4,5}$ These costs are secondary to increased use of antibiotics and longer mechanical ventilation ICU stay. Therefore, VAP prevention can reduce both the cost and morbidity associated with mechanical ventilation.

See the Related Editorial on Page 1704

Aspiration of colonized oropharyngeal secretions is one of the primary factors implicated in the pathogenesis of VAP. VAP prevention efforts have centered on reducing the bacterial load of aspirated secretions, and reducing the amount of secretions aspirated. ${ }^{6}$ With regard to the former, disinfection of the oropharynx with chlorhexidine, coupled with increased attention to oral care, has been shown to reduce the risk of VAP. ${ }^{7}$ Similarly, elevation of the head of the bed has been demonstrated to reduce the risk of 
aspiration of oropharyngeal secretions into the lung in intubated, mechanically ventilated patients and has become a nearly universally applied element to prevent VAP. ${ }^{8,9}$ Applying each of these interventions as a "bundle" has resulted in marked reduction in the incidence of VAP. ${ }^{10,11}$ However, despite these measures, VAP remains an important problem, with a reported incidence of approximately 2 cases per 1,000 ventilator days. ${ }^{12}$

Oropharyngeal secretions pool above the cuff of the endotracheal tube (ETT) and can be aspirated into the trachea despite the presence of the cuff, and may represent an important pathway for colonized secretions to enter the distal airway. Recently, modifications to the design of the ETT cuff have been the focus of interventions to reduce the risk of aspiration around the cuff, and thus reduce the risk of VAP. Employing a tapered cuff, as opposed to the standard barrel-shaped cuff, has been proposed to provide a better tracheal seal and reduce the passage of potentially contaminated secretions around the cuff and into the lung. A recent trial employed methylene blue instilled above the ETT cuff in pigs undergoing abdominal surgery. Significantly less methylene blue staining of mucosa following surgery was observed in pigs intubated with a tapered-cuff ETT, compared with those intubated with a standard barrel-shaped-cuff ETT. ${ }^{13}$ A tapered-cuff ETT (TaperGuard, Covidien, Mansfield, Massachusetts) has been approved by the FDA, and in vitro and in vivo studies have demonstrated reduced aspiration around the cuff. ${ }^{1}$ Whether these properties are efficacious in reducing VAP is not known. These specialized ETTs are more expensive than standard ETTs, but represent a "passive" intervention to reduce VAP; they require little to no additional effort on the part of care providers. As a component of institutional efforts to evaluate VAP-prevention measures, we performed a sequential observational study to test the hypothesis that hospital-wide use of a tapered-cuff ETT would result in a significant reduction of the VAP rate (VAPs/ 1,000 ventilator days).

\section{Methods}

\section{Study Design}

This 2-period observational study was approved by our institutional review board, which waived the requirement of informed consent. Each study period was 6 months in duration, and included the same calendar months, to minimize the impact of seasonal variation on incidence of VAP. A transition period, during which the hospital supply of ETTs was changed, was not included in the analysis.

\section{Inclusion Criteria}

All patients over the age of 18 admitted to any of our adult ICUs and who required endotracheal intubation and

\section{QUICK LOOK}

\section{Current knowledge}

Leakage of colonized oropharyngeal secretions around the endotracheal tube cuff is a major factor in the pathogenesis of ventilator-associated pneumonia (VAP). A tapered-cuff endotracheal tube reduces the leakage in a bench model, but the tapered cuff's ability to alter the incidence of VAP is not known.

\section{What this paper contributes to our knowledge}

In an ICU with a VAP rate near the average of United States ICUs, and where there was high adherence to a VAP prevention bundle, the tapered-cuff endotracheal tube was not associated with a reduction in the VAP rate.

mechanical ventilation at any time during their ICU stay were included. These 110 ICU beds encompassed multiple specialties, including medical ICU, surgical ICU, neurointensive care, cardiothoracic ICU, trauma ICU, and coronary care. The VAP prevention bundle and VAP data collection were identical across all ICUs.

\section{Study Procedure}

During the baseline 6 month period, July 1, 2010, through December 31, 2010, a standard barrel-shaped-cuff ETT (Mallinckrodt Hi-Lo, Covidien, Mansfield, Massachusetts) was used hospital wide. All ETTs throughout the hospital (including the operating rooms) were then replaced with a tapered-cuff ETT (TaperGuard, Covidien, Mansfield, Massachusetts). This was performed by resource management staff who purchase and stock all supplies at the medical center. Additionally, our county (Forsyth County, North Carolina) emergency medical response units were supplied with the tapered-cuff ETTs, but intubated patients transferred from other institutions were not reintubated with a tapered-cuff ETT; however, these patients were included in the study. After a hospital-wide audit to ensure removal of the barrel-shaped-cuff ETTs, the second period of the study commenced on July 1, 2011 and extended through December 31, 2011. The tapered-cuff ETT itself is of the same materials and design as the barrelshaped-cuff ETT: the difference is solely in the cuff design. This ETT does not have an extra lumen to permit removal of supraglottic secretions, and, other than the shape of the cuff when inflated, is identical in appearance to the standard barrel-shaped-cuff ETT. Therefore, no staff education concerning the replacement ETT was felt to be necessary, though it was announced that a new type of 
ETT was being studied. Respiratory therapists' procedures for caring for ETTs were unchanged throughout the study period. The respiratory therapists checked and documented the position of the ETT and maintained ETT cuff pressure at $20-25 \mathrm{~cm} \mathrm{H}_{2} \mathrm{O}$ twice during each 12 hour shift.

\section{Data Collection and Definition of VAP}

Infection Control practitioners trained in data abstraction and reporting were responsible for capturing all instances of VAP during ICU admission. They were not involved in direct patient care, and each suspected case of VAP was reviewed by the hospital epidemiologist, a board certified specialist in infectious diseases. These individuals did not change during the study period. Criteria for the diagnosis of VAP were concordant with National Healthcare Safety Network VAP criteria (PNU2), which requires bacteriologic confirmation in addition to meeting specific clinical criteria, including, for example, new radiographic infiltrates, fever, and leukocytosis. ${ }^{14}$ However, in our institution, outside the trauma ICU, instead of the bronchoscopic cultures specified in PNU2, we employ quantitatively cultured tracheal aspirate, and we use a threshold of $\geq 10^{5}$ colony-forming units (CFUs) per mL to confirm a VAP diagnosis. Within the trauma ICU, bronchoscopic and non-bronchoscopic bronchoalveolar lavage cultures were used, with a threshold of $10^{4} \mathrm{CFU} / \mathrm{mL}$ to confirm VAP. This was consistent throughout the study.

Personnel from our quality resource center monitored adherence to the institutional VAP prevention bundle and days of mechanical ventilation. These individuals had no role in the provision of clinical care. These data were collected in all ICU patients, Monday through Friday, prospectively, by chart review and bedside inspection. Data on weekends and holidays were collected retrospectively by chart review. The institutional VAP prevention bundle consisted of elevation of the head of bed to $30^{\circ}$, swabbing all oral surfaces (buccal, pharyngeal, gingival, tongue, and tooth surfaces) for 30 seconds with $15 \mathrm{~mL} 0.12 \%$ chlorhexidine gluconate twice daily, brushing teeth twice daily, general oral care every 4 hours, and orogastric in preference to nasogastric tube. VAP bundle adherence for each intubated subject was recorded daily as adherence to the entire bundle (ie, failure to perform a single component was defined as failure to adhere to the bundle for that ventilator day). Subjects in whom there was a documented medical contraindication to a component of the bundle (eg, a nasogastric tube inserted operatively in a subject undergoing esophageal surgery) were not recorded as nonadherent, provided they were adherent to all other bundle components.
Table. Subjects, Ventilator Days, and VAP Data

\begin{tabular}{lccc}
\hline \hline & $\begin{array}{c}\text { Standard-Cuff } \\
\text { Group }\end{array}$ & $\begin{array}{c}\text { Tapered-Cuff } \\
\text { Group }\end{array}$ & $P$ \\
\hline Ventilated subjects & 1,387 & 1,462 & .13 \\
Ventilator days & 7,266 & 7,984 & .20 \\
VAPs & 24 & 21 & .71 \\
VAP/1,000 ventilator days, & $3.29 \pm 1.79$ & $2.77 \pm 2.00$ & .65 \\
$\quad$ VAPs/month & $96.5 \pm 2.7$ & $90.3 \pm 3.5$ & .01 \\
VAP bundle adherence, & & & \\
$\quad$ percent adherence/month & & & \\
& & & \\
\hline values are mean \pm SD. & & & \\
VAP $=$ ventilator-associated pneumonia & & & \\
\end{tabular}

The number of intubated subjects and patient days of mechanical ventilation were recorded, and the VAP rate is expressed as VAPs per 1,000 ventilator days. A day of mechanical ventilation was defined as mechanical ventilation at any time on that calendar day.

\section{Role of the Sponsor}

This was an investigator initiated study. The sponsor (Covidien, Mansfield, Massachusetts) provided the tapered-cuff ETTs at a discounted price and provided salary support for data collection. The sponsor was not involved in study design, data collection, or data analysis. The sponsor had no access to study data, nor any role in formulating the conclusions for this report, but was allowed to review the manuscript prior to submission for publication.

\section{Statistical Analysis}

Data were entered into a secure, password-protected database, and analyzed with statistics software (SPSS 11, SPSS, Chicago, Illinois). Results were analyzed initially using descriptive statistics. Comparisons between groups were done using chi-square test for proportions, and the unpaired Student $t$ test for continuous variables. A 2-tailed $P$ value $<.05$ was considered statistically significant. From December of 2009 to June 2010, our institutional monthly VAP rate was $5.8 \pm 1.47$ per 1,000 ventilator days. We estimated that a 6-month trial period would have an $80 \%$ power to detect a $50 \%$ reduction in the primary outcome of VAPs/1,000 ventilator days, with an alpha of .05 .

\section{Results}

We included 2,849 subjects, encompassing 15,250 ventilator days. The distribution of ventilator days was $41 \%$ 


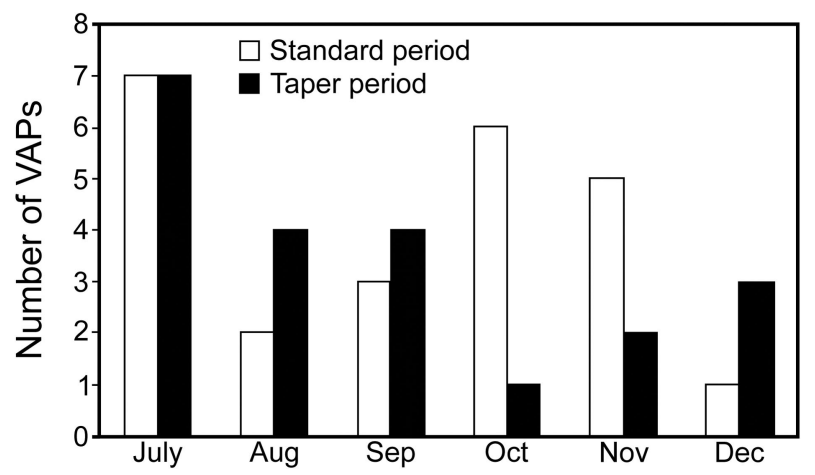

Fig. 1. Number of ventilator-associated pneumonias (VAPs) during the 2 study periods.

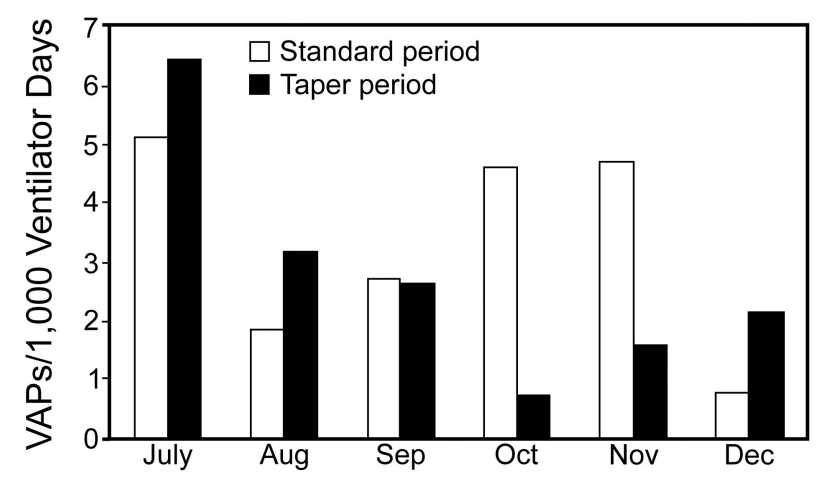

Fig. 2. Ventilator-associated pneumonia (VAP) rate (VAPs/1,000 ventilator days) during the 2 study periods.

medical ICU, 35\% surgical and trauma ICU, $11 \%$ cardiothoracic ICU, $10 \%$ neurological ICU, and 3\% other ICUs. The distribution of subjects across the ICUs did not differ between the study periods. There were 24 VAPs while using the barrel-shaped-cuff ETT (standard-cuff group), and 21 VAPs while using the tapered-cuff ETT (taperedcuff group) $(P=.71)$ (Table). The mean \pm SD monthly VAP rate was $3.29 \pm 1.79 / 1,000$ ventilator days in the standard-cuff group, and $2.77 \pm 2.00 / 1,000$ ventilator days in the tapered-cuff group $(P=.65)$.

Adherence to the VAP prevention bundle was high throughout the study period. However, VAP bundle adherence was significantly higher during the standard-cuff period $(96.5 \pm 2.7 \%)$ than in the tapered-cuff period $(90.3 \pm 3.5 \%, P=.01)$. The most common reason for VAP bundle non-adherence was failure to perform (or document) every scheduled provision of oral care. This accounted for $76 \%$ of all VAP bundle non-adherence. The number of VAPs during each study month, the monthly VAP rate, and VAP bundle adherence are displayed in Figures 1,2, and 3, respectively. There were no significant differences in the number of mechanically ventilated subjects or the number of ventilated days (patient days of mechanical ventilation) between periods.

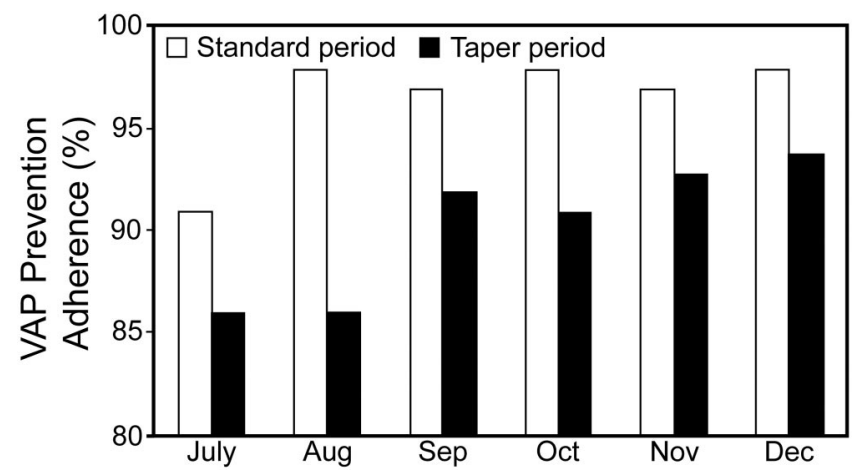

Fig. 3. Adherence to the ventilator-associated pneumonia (VAP) prevention bundle during the 2 study periods.

\section{Discussion}

The use of the tapered-cuff ETT did not significantly reduce the VAP rate. Our adherence to VAP-prevention measures was high, but it was significantly higher in the standard-cuff period than in the tapered-cuff period. The reason for this difference is not clear, but the consistency of performance of interventions to improve quality has been reported to wane over time if efforts directed at maintaining performance are not sustained. ${ }^{15}$ It is possible that reduced adherence to the VAP prevention bundle masked a significant reduction in VAP in the tapered-cuff period. However, adherence rates of $80-90 \%$, as observed in the tapered-cuff period, have been reported to still be associated with a reduced VAP rate. ${ }^{8}$ Further, an analysis of our VAP rate and VAP bundle adherence from July 2008 through June 2010 found only a weak correlation between VAP bundle adherence and VAP incidence $\left(r^{2}=0.11\right)$ (unpublished data). Collectively, while this lessens the probability, it does not preclude that the observed difference in VAP bundle adherence obscured a clinically relevant reduction in VAP attributable to the use of a tapered-cuff ETT.

The VAP rate range in the present study, 2.77-3.29/ 1,000 ventilator days, was much lower than that in other studies reporting successful interventions to reduce VAP, including elevation of the head of the bed, chlorhexidine oral rinsing, silver-coated ETTs, and subglottic secretion removal, in which VAP rates of $10-15 / 1,000$ ventilator days or higher were observed. ${ }^{16-19}$ Whether significant reductions in VAP consequent to use of a tapered-cuff ETT might be observed in settings with a higher baseline VAP rate cannot be determined from the present study. Importantly, however, the rates reported in the present study closely mirror the median rates reported by the National Healthcare Safety Network for 2010 (range approximately $0-5 / 1,000$ ventilator days, depending upon the type of ICU). ${ }^{12}$ Several studies have suggested that publicly reported VAP rates may not accurately reflect 


\section{The Impact of Hospital-Wide Use of a Tapered-Cuff Endotracheal Tube}

clinical VAP. ${ }^{20,21}$ However, a study of patients in our institution indicated that, while specific patients may differ, the rates of pneumonia were similar whether bronchoscopic or National Healthcare Safety Network criteria were used.22

While this study was designed to be powered to detect a $50 \%$ reduction in the VAP rate, this was predicated on a historical VAP rate of $5.8 \pm 1.47$ per 1,000 ventilator days, present in early 2010 during study planning. The observed baseline rate was only 3.29/1,000 ventilator days. With this baseline VAP rate a study duration of 3 years would be required to have an $80 \%$ power to detect a $50 \%$ reduction. This underscores the difficulty of executing adequately powered studies of VAP prevention in the setting of successful baseline efforts to reduce the VAP rate.

Of note, in contrast to the bronchoscopic quantitative cultures used in the National Healthcare Safety Network PNU2 definition of VAP, quantitative tracheal aspirates with a threshold of $10^{5} \mathrm{CFU} / \mathrm{mL}$ were employed to confirm VAP. However, this criterion has been shown to result in a VAP rate virtually identical to the rate found if using a bronchoalveolar lavage quantitative culture threshold of $10^{4} \mathrm{CFU} / \mathrm{mL} .^{23,24}$

Another limitation of this study is that transfer patients were not re-intubated with a tapered-cuff ETT. Patient referral and transfer patterns in our institution were not known to change over the study period, and, while unlikely, an unappreciated increase in these patients with an attendant higher VAP incidence may have masked a decrease in VAP in subjects intubated with the taperedcuff ETT. While all mechanically ventilated adult patients were included, the limited data elements collected for the study precludes ascertainment of the impact of timerelated changes in the demographic characteristics, severity of illness, or comorbidities on the incidence of VAP.

Only VAP occurring in an ICU was captured. However, all patients who were mechanically ventilated were initially cared for in an ICU, and only a small minority are ultimately transferred to a step-down unit for longer-term ventilator management. Only one VAP was observed in the step-down unit during the study period. Thus, comparison of the VAP rates in the standard-cuff and tapered-cuff groups should not be affected by this limitation.

\section{Conclusions}

In the setting of a VAP rate very near the average of ICUs in the United States, and where there was high adherence to a VAP prevention bundle, the use of a taperedcuff ETT was not associated with a significant reduction in the VAP rate.

\section{REFERENCES}

1. Lorente L, Blot S, Rello J. New issues and controversies in the prevention of ventilator-associated pneumonia. Am J Respir Crit Care Med 2010;182(7):870-876.

2. Porzecanski I, Bowton DL. Diagnosis and treatment of ventilatorassociated pneumonia. Chest 2006;130(2):597-604.

3. Tejerina E, Frutos-Vivar F, Restrepo MI, Anzueto A, Abroug F, Palizas F, et al. Incidence, risk factors, and outcome of ventilatorassociated pneumonia. J Crit Care 2006;21(1):56-65.

4. Restrepo MI, Anzueto A, Arroliga AC, Afessa B, Atkinson MJ, Ho $\mathrm{NJ}$, et al. Economic burden of ventilator-associated pneumonia based on total resource utilization. Infect Control Hosp Epidemiol 2010; 31(5):509-515.

5. Warren DK, Shukla SJ, Olsen MA, Kollef MH, Hollenbeak CS, Cox MJ, et al. Outcome and attributable cost of ventilator-associated pneumonia among intensive care unit patients in a suburban medical center. Crit Care Med 2003;31(5):1312-1317.

6. Gastmeier P, Geffers C. Prevention of ventilator-associated pneumonia: analysis of studies published since 2004. J Hosp Infect 2007; 67(1):1-8

7. Labeau SO, Van de Vyver K, Brusselaers N, Vogelaers D, Blot SI. Prevention of ventilator-associated pneumonia with oral antiseptics: a systematic review and meta-analysis. Lancet Infect Dis 2011;11(11): 845-854.

8. Bird D, Zambuto A, O'Donnell C, Silva J, Korn C, Burke R, et al. Adherence to ventilator-associated pneumonia bundle and incidence of ventilator-associated pneumonia in the surgical intensive care unit. Arch Surg 2010;145(5):465-470.

9. Bouadma L, Mourvillier B, Deiler V, Le Corre B, Lolom I, Régnier $\mathrm{B}$, et al. A multifaceted program to prevent ventilator-associated pneumonia: impact on compliance with preventive measures. Crit Care Med 2010;38(3):789-796.

10. Bouadma L, Deslandes E, Lolom I, Le Corre B, Mourvillier B, Régnier B, et al. Long-term impact of a multifaceted prevention program on ventilator-associated pneumonia in a medical intensive care unit. Clin Infect Dis 2010;51(10):1115-1122.

11. Morris AC, Hay AW, Swann DG, Everingham K, McCulloch C, McNulty J, et al. Reducing ventilator-associated pneumonia in intensive care: impact of implementing a care bundle. Crit Care Med 2011;39(10):2218-2224.

12. Dudeck MA, Horan TC, Peterson KD, Allen-Bridson K, Morrell G, Pollock DA, Edwards JR. National Healthcare Safety Network (NHSN) report, data summary for 2010, device-associated module. Am J Infect Control 2011;39(10):798-816.

13. Lichtenthal PR, Maul D, Borg U. Do tracheal tubes prevent microaspiration? Brit J Anaesth 2011;107(5):821-822.

14. Horan TC, Andrus M, Dudeck MA. CDC/NHSN surveillance definition of health care-associated infection and criteria for specific types of infections in the acute care setting. Am J Infect Control 2008;36(5):309-332.

15. Ely EW, Bennett PA, Bowton DL, Murphy SM, Florance AM, Haponik EF. Large scale implementation of a respiratory therapistdriven protocol for ventilator weaning. Am J Respir Crit Care Med 1999;159(2):439-446.

16. Kollef MH, Afessa B, Anzueto A, Veremakis C, Kerr KM, Margolis $\mathrm{BD}$, et al; NASCENT Investigation Group. Silver-coated endotracheal tubes and incidence of ventilator-associated pneumonia. The NASCENT randomized trial. JAMA 2008;300(7):805-813

17. Genuit T, Bochicchio G, Napolitano LM, McCarter RJ, Roghman MC. Prophylactic chlorhexidine oral rinse decreases ventilatorassociated pneumonia in surgical ICU patients. Surg Infect (Larchmt) 2001;2(1):5-18. 
18. Smulders K, van der Hoeven H, Weers-Pothoff I, VandenbrouckeGrauls C. A randomized clinical trial of intermittent subglottic secretion drainage in patients receiving mechanical ventilation. Chest 2002;121(3):858-862.

19. Drakulovic MB, Torres A, Bauer TT, Nicolas JM, Nogue S, Ferrer M. Supine body position as a risk factor for nosocomial pneumonia in mechanically ventilated patients: a randomised trial. Lancet 1999; 354(9193):1851-1858.

20. Thomas BW, Maxwell RA, Dart BW, Hartmann EH, Bates DL, Mejia VA, et al. Errors in administrative-reported ventilator-associated pneumonia rates: are never events really so? Am Surg 2011;77(8):998-1002.

21. Morris AC, Kefala K, Simpson AJ, Wilkinson TS, Everingham K, Kerslake D, et al. Evaluation of the effect of diagnostic methodology on the reported incidence of ventilator-associated pneumonia. Thorax 2009;64(6):516-522.

22. Miller PR, Johnson JC, III, Karchmer T, Hoth JJ, Meredith JW, Chang MC. National nosocomial infection surveillance system: from benchmark to bedside in trauma patients. J Trauma 2006;60(1): 98-103.

23. Wu CL, Yang DI, Wang NY, Kuo HT, Chen PZ. Quantitative culture of endotracheal aspirates in the diagnosis of ventilator associated pneumonia in patients with treatment failure. Chest 2002;122(2):662-668.

24. Mondi MM, Chang MC, Bowton DL, Kilgo PD, Meredith JW, Miller PR. Prospective comparison of bronchoalveolar lavage and quantitative deep tracheal aspirate in the diagnosis of ventilator associated pneumonia. J Trauma 2005;59(4):891-896.

This article is approved for Continuing Respiratory Care Education credit. For information and to obtain your CRCE

(free to AARC members) visit 